Meta

Journal des tradlucteurs

Translators' Journal

CHARAUDEAU, Patrick (1992) : Grammaire du sens et de

l'expression, Paris, Hachette Éducation, 927 p.

\title{
Richard Patry
}

Volume 40, numéro 1, mars 1995

URI : https://id.erudit.org/iderudit/003796ar

DOI : https://doi.org/10.7202/003796ar

Aller au sommaire du numéro

Éditeur(s)

Les Presses de l'Université de Montréal

ISSN

0026-0452 (imprimé)

1492-1421 (numérique)

Découvrir la revue

Citer ce compte rendu

Patry, R. (1995). Compte rendu de [CHARAUDEAU, Patrick (1992) : Grammaire du sens et de l'expression, Paris, Hachette Éducation, 927 p.] Meta, 40(1),

153-159. https://doi.org/10.7202/003796ar

Ce document est protégé par la loi sur le droit d'auteur. L'utilisation des services d’Érudit (y compris la reproduction) est assujettie à sa politique d'utilisation que vous pouvez consulter en ligne.

https://apropos.erudit.org/fr/usagers/politique-dutilisation/
Cet article est diffusé et préservé par Érudit.

Érudit est un consortium interuniversitaire sans but lucratif composé de l’Université de Montréal, l’Université Laval et l’Université du Québec à Montréal. Il a pour mission la promotion et la valorisation de la recherche. https://www.erudit.org/fr/ 
CHARAUDEAU, Patrick (1992): Grammaire du sens et de l'expression, Paris, Hachette Éducation. $927 \mathrm{p}$.

«Le langage est ce matériau qui permet à l'homme de construire du sens dans le monde tout en entrant en communication avec les autres. Le langage est a la fois sens, expression et communication. Il n'est pas l'un et l'autre successivement, il est les trois a la fois.» (p. 4)

Une grammaire du français de plus sur les rayons des librairies. Il s'en trouvera sans doute plusieurs qui n'auront répondu que par un haussement d'épaules désabusé à l'annonce de cette nouvelle parution, et de manière générale on se demandera certainement qu'estce que cette grammaire a à offrir de plus que les autres récemment parues, au demeurant fort nombreuses, comme par exemple celles de Jean David, de Joëlle Gardes-Tamine, de Jean Dubois ou de Michel Arrivél.

En fait, l'examen de cette problématique du «positionnement» de la Grammaire du sens et de l'expression (GSE) dans la tradition de l'étude grammaticale du français, qui sera l'objet du développement suivant, constitue une condition indispensable, autant pour l'évaluation générale de la portée et de la pertinence de l'ouvrage que pour une appréciation adéquate de son organisation et de son contenu (qui seront présentés suite à l'examen de la première question).

\section{LA GSE ET LA GRAMMAIRE DU FRANÇAIS}

L'ouvrage qui nous est proposé par Patrick Charaudeau offre-t-il quelque chose de «plus» que ceux actuellement disponibles, je ne saurais le dire. Cependant, je puis affirmer avec certitude qu'il offre quelque chose de "différent", différence fondamentale qui se situe à la racine même de l'entreprise ; soit dans la façon d'aborder le langage comme objet de conceptualisation d'une grammaire. 
Les ouvrages actuellement disponibles ont en commun d'être des grammaires de la «langue» dont l'analyse et la catégorisation sont abordées de façon essentiellement structurale. Ces grammaires présentent de façon progressive l'étude des différents niveaux de structuration internes de la langue en partant généralement de la prononciation, pour passer ensuite à la formation des mots, à l'étude des parties du discours (ou catégories grammaticales) pour terminer par l'organisation de base de la proposition et de la phrase, le tout étant présenté de façon rigoureuse et systématique de façon à permettre une consultation rapide et efficace de ces documents qui sont le plus souvent asse $z$ volumineux.

En manière de boutade, il est possible de situer globalement la GSE en disant qu'elle intègre au projet de description grammaticale "l'avant" et "l'après" du matériau verbal lui-même : soit la mise en scène situationnelle qui donne lieu à son utilisation, et l'acte de communication sur lequel cette utilisation débouche inévitablement?

En fait. l'ouvrage de Patrick Charaudeau se démarque des autres grammaires du français par une différence de perspective majeure qu'il est nécessaire d'expliciter avant de passer à la présentation de son organisation et de son contenu.

\section{LA COMMUNICATION ET NON LA L.ANGUE:}

L'objet de la GSE n'est pas la langue pour elle-même, mais la ucommunication», et cette différence de perspective est attribuable à la position de l'auteur sur l'analyse du langage, position dont l'expression se retrouve dans le modèle d'analyse asociocommunicatif' qu'il a progressivement élaboré au cours de la dernière décennie ${ }^{3}$.

Ce modèle sociocommunicatif se distingue particulièrement par la prise en considération conjointe et simultanée des espaces "interne" et "externe" dans l'analyse des faits langagiers; espace "interme" dans lequel le langage est perçu comme transparent et référentiel, et les êtres de parole comme des créatures désincarnées dont on cherche essentiellement à rendre compte des ressemblances, et espace "externe' dans lequel le langage est perçu comme étant opaque et les êtres de parole dans ce qui les individualise et souvent dans ce qui les oppose.

Pour l'auteur, ces deux approches de l'analyse du langage. qui ont connu et connaissent encore des développements distincts dans la linguistique contemporaine, ne représentent pas des alternatives correspondant à des parcours théoriques et des choix méthodologiques différents, mais constituent bien les deux assises fondamentales d'une perspective de l'analyse du langage qui soit véritablement intégrale et féconde. L'univers référentiel et les intentions communicatives doivent être appréhendés au sein d'un même modèle puisque l'un ne peut être construit qu'à travers l'autre. Finalement, cette appréhension simultanée ne peut se satisfaire des termes d une relation additive ou de complémentarité, mais doit reposer sur une intégration véritable. Les intentions ne sont pas "l'après coup" ou un épiphénomène de la référence, elles sont constitutives de la construction de cette dernière: «Le monde n’est pas donné au départ. Il se fait à travers la stratégie humaine de signification." $(198.3: 14)^{5}$.

L'intégration de ces deux espaces amène l'auteur à analyser les faits langagiers dans la situation communicative où ils ont lieus. Cependant, à la différence de plusieurs autres approches. cette situation $n^{\prime}$ 'a pas ici qu une fonction accessoire ou utilitaire, elle constitue, au contraire, un espace pleinement assumé, qui est le point de départ de l'analyse des faits langagiers dans une modélisation comprenant quatre composantes principales qui sont les suivantes:

Situation de communication

Composante de loordre du psycho-social qui est externe au langage tout en y participant. Lieu où se construit un contrat d'échange langagier entre les partenaires et où sont prises en considération l'identité et les intentions communicatives des partenaires. 
Modes d'organisation du discours

Cette composante comprend les procédés de mise en scène de l'acte de communication qui correspondent à certaines finalités. Ces procédés sont: L'ÉNONCIATION, LA DESCRIPTION, L.A NARRATION ET L.'ARGUMENTATION.

\section{Catégories de la langue}

Ces dernières sont d'ordre strictement linguistique et organisent les signes en systèmes formels signifiants.

Texte

Il s’agit ici du produit-résultat de l'acte de communication qui est fabriqué avec de la langue et du discours, mais qui est de nature différente de ces deux demiers.

Au contraire des ouvrages disponibles, dont l'analyse se concentre sur l'unique composante des "catégories de la langue», celui de Patrick Charaudeau prend comme point de départ une acception plus généreuse du phénomène langagier, el qui est surtout plus conforme aux pratiques langagières effectives des locuteurs du français. L'objet de la GSE consiste donc en une intégration des quatre composantes présentées précédemment : soit le sens communiqué d'un texte réalisé par l'intermédiaire d'une mise en forme des catégories de la langue dans un mode d'organisation du discours en fonction du projet de parole actualisé dans un cadre situationnel donné.

Comment construit-on une grammaire à partir d'une telle acception de l'analyse du langage? Un premier élément de réponse est donné explicitement par l'auteur dans les trois objectifs principaux qu'il fixe à son entreprise :

Une grammaire du sens et de l'expression doit donc s'intéresser à décrire les faits langagiers en fonction:

- des intentions du sujes parlant qu'ils sont susceptibles d'exprimer. ce qui exige que les catégories de la langue soient regroupées autour de ces intentions:

- des enjeux communicatifs qu'ils révèlent, ce qui exige que les différents systèmes de la langue soient traités du point de vue du sens:

- des effets de discours qu'ils peuvent produire, ce qui exige que soient passés en revue les différents types d'usages vivants de la langue, et pas seulement les usages littéraires. (p. 4)

À l'extension «étendue» des phénomènes langagiers pris en considération viennent donc s'ajouter des objectifs spécifiques du point de vue de la portée de l'analyse et du regroupement des faits de langage pris en considération. Ces dimensions originales et novatrices de la GSE ne peuvent être explicitées davantage que par un examen de l'organisation et du contenu de cet ouvrage, qui n'a pas de "grammaire" que le nom, mais qui constitue un manuel de référence au plein sens du terme et qui ne cède en rien en matière de rigueur et de systématicité aux ouvrages plus traditionnels.

\section{LA GRAMMAIRE DU SENS ET DE L'EXPRESSION : ORGANISATION ET CONTENU}

La GSE comprend trois parties principales qui seront présentées en détail et qui sont les suivantes: (1) les mécanismes du sens el la construction des mots, (2) les catégories de la langue, et finalement (3) les modes d"organisation du discours.

\section{Les mecanismes du sens et la construction des mots}

Cette première partie, qui est la moins étendue de l'ouvrage (pp. 11-116), constitue un développement introductif sur l'analyse du signe linguistique qui est divisé en cinq parties principales. 
Dans la première partie qui se nomme "(Généralités", l'auteur introduit les notions de base de l'analyse du signe linguistique: soit les principaux concepts opératoires découlant de la distinction entre la forme (signifiant) et le sens (signifié) des mots. L'exposé de ces notions fondamentales permet ensuite l'introduction de la deuxième partie qui s"intitule "Nommer». Dans celte dernière, l'autcur amorce sa réanalyse sémantique des parties du discours en regroupant les catégories grammaticales de classes ouvertes dans les trois "classes conceptuclles" suivantes qu'il analyse par la suite successivement du point de vue de leurs caractéristiques sémantiques et formelles respectives: les fitres (le substantif), les Processus (le verbe) et les Propritétés (les adjectifs et les adverbes). Le troisième développement se nomme "Classer" et consiste en une présentation de base de la structure d'oppositions sémantiques selon laquelle s'organise le lexique de classes ouvertes du vocabulaire du français. Ce développement introduit les principales relations lexico-sémantiques paradigmatiques et les mécanismess de la combinatoire sur l'axe syntagmatique. La quatrième partic s'intitule "Construire" et présente les principaux mécanismes de formation des mots en français (préfixation. suffixation. composition). les voies par lesquelles s'effectue l'apport de vocabulaire ou l'introduction de nouveaux mots (ellipse, troncation, sigles, emprunts) et les mécanismes rhétoriques de transfert de sens (hyperbole, ironie, litote, métaphorisation). Finalement, le dernier développement de celte partie se nomme "Valeur sociale du signe" et consiste en l'introduction de notions telles l'identité du locuteur, de sociolectes, de marché linguistique et de valeur sociale du signe qu'il est déterminant de prendre en considération dans la conception de tout projet de grammaire, el plus particulièrement lorsque celle-ci a pour cible principale le sens et lexpression.

\section{Les catégories de la langue}

Il s'agit ici du développement de loin le plus élaboré de l'ouvrage (pp. 119-63.3) et auquel il est le plus difficile de rendre justice dans ce compte rendu, tant en raison de son étendue que de la richesse de détail des analyses qui y sont proposées.

Cette deuxième partie de la GSE comprend donc globalement une présentation des différentes parties du discours. Cependant, ces dernières sont présentées dans un système à double entrée (titre de section comprenant deux notions clés) permettant à l'auteur à la fois de s"inscrire dans la continuité de la tradition des grammaires du français et de réaliser l'une des dimensions fondatrices de son projet: à savoir d'organiser la description des faits analysés sur un axe prioritairement «sémantique" et non "catégoricl». Cette double mise en forme de l'analyse des catégories de la langue donne donc le sommaire suivant pour celte deuxième partic:

\author{
«axe sémantique» \\ La personne \\ L'actualisation \\ La dépendance \\ La désignation \\ La quantification \\ L'identification indéterminée \\ La présentation \\ La qualification, la comparaison \\ Laction \\ La localisation dans l'espace \\ La situation dans le temps ${ }^{\text {h }}$ \\ L'argumentation \\ L'affirmation
}

\section{*catégories de la langue»}

et les pronoms personnels

et l'article

et les possessifs

et les démonstratifs

et les quantificateurs

et les indéfinis

et les présentateurs

et les procédés d'adjectivation

et les actants

et les relations logiques?

et la négation 
Chacune de ces rubriques est développée par l'auteur de façon systématique en quatre points majeurs.

Le premier développement nommé “Généralités" présente une mise au point critique du thème de la rubrique traitée autant du point de vue grammatical que linguistique. Par exemple, pour la première rubrique qui est «La personne et le pronom personnel», l'auteur observe que dans les ouvrages grammaticaux. la catégorie des upronoms personnels» est généralement présentée avec les autres pronoms (possessifs, démonstratifs, indéfinis) et qu'elle est introduite comme une catégorie monolithique et homogène. Face à cet état de fait. il fait valoir que tous les pronoms ne satisfont pas aux conditions de la même opération sémantique et que du point de vue de "la personne" il est préférable de considérer les pronoms personnels séparément. En second lieu, il insiste sur le fait que les pronoms personnels ne sont pas tous identiques et qu'il est plus fructueux sur le plan de la description de distinguer les marques pronominales «élocutives» (je/me/moi), «allocutives» (tu/te/toi) et "délocutives» (il/ils/eux).

Le second développement nommé «Marques et formes» présente une description analytique détaillée de chacun des mots compris dans les différents niveaux de structuration d'une rubrique donnée (exemple: chaque forme pronominale de chacune des trois catégories de pronoms dans la rubrique aLa personne et les pronoms personnels"). Le troisième. intitulé «Particularités sémantiques». présente la valeur sémantique fondamentale de chacun des différents niveaux de structuration d'une rubrique donnée (exemple : valeur de désignation des marques pronominales élocutives, d'interlocution des marques allocutives et valeur anaphorique des marques délocutives). Ces valeurs fondamentales sont évidemment illustrées de nombreux exemples mettant en évidence les catégories de la langue intéressées par un développement donné. Finalement, le demier développement, nommé «Faits de discours", présente les différents effets de sens possibles des éléments d'une rubrique donnée en fonction des différents types de contextes et de situations de communication dans lesquelles elles peuvent être employées\$. Par exemple, dans le cas des pronoms personnels, l'auteur s'intéresse aux effets de discours produits par la combinaison des différents pronoms avec certains prédicals verbaux, aux substitutions de personnes, et aux effets observables pour cette catégorie de la langue dans différents types de textes (scientifiques, didactiques, propagande politique, littéraires).

Cette deuxième partie, la plus étendue et la plus élaborée de l'ouvrage, est celle qui permet le mieux de saisir l'essence même du projet de description grammaticale de Patrick Charaudeau, celle où il ne s'enracine dans la description selon l'organisation catégorielle que pour mieux s'en écarter. et présenter les faits de langage d'un point de vue résolument sémantique et communicatif.

\section{Modes d'organisation du discours}

Cette troisième et demière partie, d'une étendue se situant entre celles des deux autres (pp. 6.33-8.35), est certes la plus originale et la plus novatrice de l'ouvrage et est sans équivalent dans les autres grammaires du français. Son objet principal consiste en l'actualisation des catégories de la langue à des fins d'expression et de communication dans les quatre formes fondamentales de mise en discours distinguées par l'auteur, ces dernières, qui sont les suivantes. étant d'emblée caractérisées par la dominance d'une opération sémantique :

«Formes de mise en discours»
Énonciation
Description
Narration
Argumentation

\author{
«Opérations sémantiques» \\ Modalisation \\ Qualification \\ Action \\ Relations logiques
}


L'auteur débute cette partie de la GSE portant spécifiquement sur l'expression et la communication par la présentation d'une synthèse des concepts clés de son modèle sociocommunicatif". Ensuite, il présente les quatre modes d'organisation du discours dont il propose respectivement une définition, une composante fonctionnelle de base, ainsi que les différents procédés linguistiques et discursifs impliqués par la mise en forme de chacun d'entre eux ${ }^{10}$.

\section{Index et références}

Cet ouvrage comprend des annexes (pp. 8.37-927) qui s'avèrent un complément d'information très utile tant comme source d'informations générales sur la langue que pour une consultation efficace et rapide de l'ouvrage. Cette dernière partie de la GSE comprend des tableaux de conjugaisons, une bibliographie sommaire pour chacune des trois parties. un index des auteurs cités, un index alphabétique général et une table des matières détaillée.

Finalement, on ne peut terminer cette présentation du contenu de la GSE sans mettre en évidence la qualité impressionnante de sa présentation matérielle.

Les divisions de chaque rubrique de l'ouvrage sont identifiées par des titres et soustitres qui reviennent systématiquement et qui sont clairement identifiés soit par la taille soit par la police de caractères utilisées (ou les deux). De plus, chaque paragraphe est numéroté, ce qui facilite la consultation des nombreux renvois (principalement dans la deuxième partie). et les différents développements de l'ourrage contiennent de nombreux tableaux récapitulatifs qui présentent de façon synthétique les concepts clés de certains développements importants ou plus élaborés. En dernier lieu, il convient d'insister sur la richesse et la variété des exemples proposés dans chaque partie de l'ouvrage qui peuvent être des extraits de textes d'auteurs (littéraires, joumalistiques, scientifiques), des slogans publicitaires, des bandes dessinées... é dont les éléments saillants dans un développement donné sont toujours mis en évidence de façon très claire.

\section{CONCLUSION}

La (irammaire du sens et de lexpression est donc une entreprise concluante, un projet audacieux dans lequel l'auteur a réussi à tenir le pari combien difficile de proposer une "véritahle" grammaire tout en s"écartant significativement des sentiers hattus et en abordant de façon substantielle le plan de la communication qui n'avait jamais été développé que de façon ponctuelle dans les autres descriptions grammaticales du français.

Cel ouvrage constitue un manuel de référence indispensable pour tous les professionnels soucieux de la relation entre la langue et ses usagers. Les enseignants, les traducteurs, les littéraires, de mème que ceux ceuvrant dans le domaine de la seciolinguistique et de la linguistique appliquée le consulteront avec plaisir et profit.

La Grammaire du sens et de l'expression apporte, d'une certaine façon, une consécration aux développements de l'analyse du discours en linguistique des vingt dernières années. et démontre de façon magistrale, par leur intégration à l'entreprise de description grammaticale, que ces développements sont non seulement «intéressants» et "pertinents". mais qu’ils peuvent également être... «utiles"s.

RICHARD PATRY

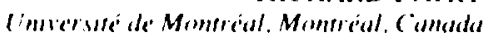

\section{Notes}

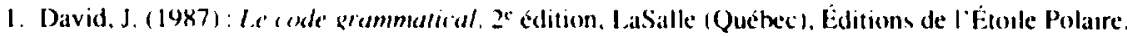

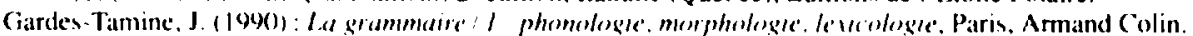

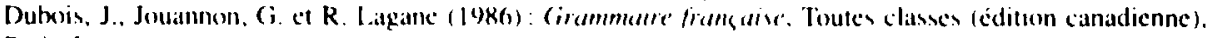
Paris, l.arousse.

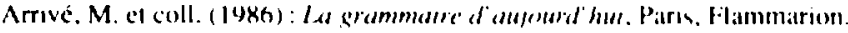


2. La question de savoir si le langage est véritablement utilisé à des fins communicatives ou si l'acte de communication est réussi ou non représentent des problématiques distinctes qui ne sont pas pertinentes dans le cadre de ce développement.

3. Une synthèse des principaux concepts de ce modèle conslitue le développement introductif de la troisième partie de la (iSE (pp. 6.34-646) intitule aLes modes d'organisation du discours".

4. Charaudeau, P. (198.3): Langage él discours. Eléments de sémishlinguistique. (Thérie el pratique), Paris. Hachette Université.

5. Pour une présentauon plus détaillée du modèle sociocommunicatif, voir également les références suivantes : Charaudeau. P. ( $1989 \mathrm{a})$ : “Le dispositif sociocommunicatif des echanges langagiers $\cdots$, Verbum, XII, pp. 13-26. Charaudeau. P. (1989b): "L a conversation entre le situationnel et le linguistique". (unnetuons, 53, pp. 9.23. Charaudeau. P. (1991): "Contrats de communication et ritualisation des débats télévisés". P. Charaudeau et coll., La télevision. Le's déhats teleivisés: "Apostrophe's., Paris, Didier Érudition, pp. I1-36.

6. Ces deux dimensions sémantiques de l'analyse du sens et de l'expression peuvent être réalisées par une trop grande variété de catégories de la langue pour qu il soit possible d'en désigner une de façon particulière.

7. Pour une etude du statut des relations logiques (ou argumentatives) dans l'analyse du discours, voir principalement

Ducrot, O. el coll. ( 1980 ) : Les mots du discours, Paris, Les Éditions de Minuit.

Roulet. E. et coll. (1985) : L'articulation du discours en fransais contemporain. Beme. Peter Lang.

8. Pour l'auteur. ces deux notions de "conleve" el de asiruallon" ne sont pas synonymes. La première fait référence à l'environnement langagier (ou textuel) d'une séquence verbale donnéc (ce que l'on nomme egalement parfois "c(o-texf(")). alors que la seconde fait reférence à des composantes de l'environnement physique de l'acte de communication: "...le Contexte est interme a l'acte de langage et toujours configuré d'une certaine façon (texte verbal, image, graphisme. etc.), alors que la Situation est exteme à l'acte de langage tout en en constituant les conditions de réalisation. m (p. 6.37)

9. Voir la première partie de ce compte rendu (voir La communication et non la langue).

10. Un résumé adéqual et representatif du contenu de cette demière partie demanderait un développement considérable dépassant les limites de ce compte rendu, c'est pourquoi je me limite ici à un développement aussi schématique. 